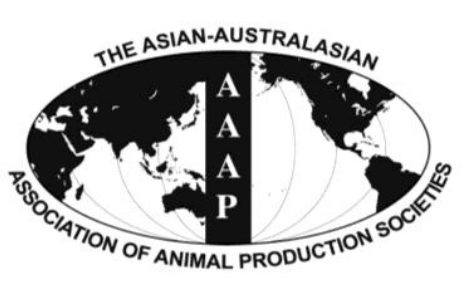

Open Access

Asian Australas. J. Anim. Sci.

Vol. 27, No. 12 : 1783-1793 December 2014

http://dx.doi.org/10.5713/ajas.2014.14720

www.ajas.info

pISSN 1011 1-2367 elSSN 1976-5517

\title{
Effects of Capsaicin on Adipogenic Differentiation in Bovine Bone Marrow Mesenchymal Stem Cell
}

\author{
Jin Young Jeong ${ }^{\text {a }}$, Sekar Suresh ${ }^{\text {a }}$, Mi Na Park, Mi Jang, Sungkwon Park ${ }^{1}$ Kuppannan Gobianand, \\ Seungkwon You' ${ }^{2}$, Sung-Heom Yeon, and Hyun-Jeong Lee* \\ Division of Animal Genomics and Bioinformatics, National Institute of Animal science, \\ Rural Development Administration, Suwon 441-706, Korea
}

\begin{abstract}
Capsaicin is a major constituent of hot chili peppers that influences lipid metabolism in animals. In this study, we explored the effects of capsaicin on adipogenic differentiation of bovine bone marrow mesenchymal stem cells (BMSCs) in a dose- and time-dependent manner. The BMSCs were treated with various concentrations of capsaicin $(0,0.1,1,5$, and $10 \mu \mathrm{M})$ for 2,4 , and 6 days. Capsaicin suppressed fat deposition significantly during adipogenic differentiation. Peroxisome proliferator-activated receptor gamma, cytosine-cytosine-adenosine-adenosine-thymidine/enhancer binding protein alpha, fatty acid binding protein 4, and stearoyl-CoA desaturase expression decreased after capsaicin treatment. We showed that the number of apoptotic cells increased in dose- and timedependent manners. Furthermore, we found that capsaicin increased the expression levels of apoptotic genes, such as B-cell lymphoma 2-associated X protein and caspase 3. Overall, capsaicin inhibits fat deposition by triggering apoptosis. (Key Words: Bovine Bone Marrow Mesenchymal Stem Cells [BMSCs], Capsaicin, Adipogenesis, Differentiation, Apoptosis)
\end{abstract}

\section{INTRODUCTION}

Excess fat deposition affects human obesity and lipid metabolism disorders. In addition, fat accumulation is an important health issue in humans and animals. Previous reports have proposed mechanisms to reduce fat accumulation through energy intake, energy expenditure, preadipocyte differentiation and proliferation, lipogenesis, lipolysis and fat oxidation (Wang and Jones, 2004; Kennedy et al., 2010). Among these, adipogenesis regulates

\footnotetext{
* Corresponding Author: Hyun-Jeong Lee. Tel: +82-31-290-1207, Fax: +82-31-290-1602, E-mail: hyunj68@korea.kr

${ }^{1}$ Animal Nutrition and Physiology Team, National Institute of Animal Science, Rural Development Administration, Suwon 441706, Korea.

2 The Laboratory of Cell Growth and Function Regulation, Division of Bioscience and Technology, College of Life and Environmental Sciences, Korea University, Seoul 136-701, Korea.

${ }^{\mathrm{a}}$ These authors contributed equally to this work.

Submitted Sept. 16, 2014; Revised Sept. 22, 2014; Accepted Sept. 29, 2014
}

preadipocyte differentiation into adipocytes. Therefore, understanding adipocyte cell differentiation may be useful to prevent lipid accumulation and improve meat quality. Adipocytes increase in number after cell differentiation, which involves changes in the size and shape of preadipocytes and transcriptional activation of adipogenesis-related genes (Wise and Green, 1979).

Fat deposition in cattle muscle is influenced by various factors, such as the breed, genotype, gender, age, and castration and nutrition statuses of cattle (Jeong et al., 2012; Moisá et al., 2013). In addition, fat deposition is a valuable economic trait in the beef industry. Various inhibitory factors suppress adipogenesis in vitro. The beef industry must reduce the fat content in meat carcasses to decrease the amount of inedible fat, while maintaining beef quality. Therefore, fat metabolic mechanisms and nutritionmodifying technologies are required to manipulate fat deposition in cattle.

Mesenchymal stem cells (MSCs) are multipotent progenitor cells that can differentiate into adipocytes (Tang and Lane, 2012 into adipocytes is). Differentiation of MSCs 
is also regulated by a variety of endocrine and paracrine factors. The MSCs have been isolated and examined from the bone marrow of many mammals, including laboratory rodents (Simmons et al., 1991), humans (Pittenger et al., 1999), and pigs (Ringe et al., 2002). Bone marrow and adipose tissue are common sources of stem cells derived from animals. Thus, bone marrow mesenchymal stem cells (BMSCs) are invaluable for therapies and as disease models in humans and cattle. These stem cells reside in the vascular stroma of adipose tissue, as well as in the bone marrow. Bone marrow may represent a source of stem cells and could be used for further studies among diverse fields (Zuk et al., 2001). Thus, adipocytes are derived from multipotent MSCs that can develop into several cell types (e.g. adipocytes, myocytes, chondrocytes, and osteocytes) (Prockop, 1997; Pittenger et al., 1999; Deans and Moseley, 2000; Woodbury et al., 2000).

Capsaicin is a major constituent of chili peppers and acts as a chemotherapeutic agent in obese models. Capsaicin induces apoptosis via a p53-dependent mechanism, through reactive oxygen species (ROS) and through the activation of caspase-3 (CASP3). In addition, capsaicin can induce apoptosis (Hsu and Yen, 2007). Therefore, fat mass and serum triglyceride levels decrease after capsaicin treatment. Furthermore, capsaicin intake can increase energy expenditure and fat oxidation by activating adipose tissue. Capsaicin was found to inhibit various signaling pathways of adipocyte differentiation via inhibition of enhancer binding protein alpha (CEBPA) and peroxisome proliferator-activated receptor gamma (PPARG) family genes (Hwang et al., 2005). The CEBPA and PPARG genes are important transcription factors involved in adipogenesis. Fatty acid binding protein 4 (FABP4) and stearoyl-CoA desaturase $(S C D)$ genes regulate PPARG target genes to induce adipocyte differentiation (Shin et al., 2009). Capsaicin inhibits the growth of various immortalized, transformed, and tumor cells (Lin et al., 2013). As described above, the effects of capsaicin have been widely studied (e.g. anti-adipogenesis, anti-tumor, and anti-proliferation).

Cell apoptosis is important for the destruction of undesired cells during development and homeostasis of multicellular organisms. There are two main pathways that lead to apoptosis: intrinsic and extrinsic. Capsaicin induces apoptosis and modulates Mitogen-activated protein kinases signaling through activation of CASP3 and phosphorylation of extracellular signal-regulated kinase (Park et al., 2014). Caspases are a family of cysteine proteases activated during the apoptotic process, which is regulated by CASP3 and associated $\mathrm{X}$ protein (BAX). The CASP3 is also an important non-apoptotic factor involved in regulation, migration, and differentiation (Zhang et al., 2013). The BAX is a pro- and anti-apoptotic member of the B-cell lymphoma 2 family that regulates apoptosis via the intrinsic pathway, whereas CASP3 regulates apoptosis via the extrinsic pathway. In our previous study, phenolic acids, such as Epigallocatechin-3-gallate, improved the inhibition of cell growth and induction of apoptosis in BMSCs. However, the effects of capsaicin on apoptosis induction and adipogenesis inhibition in BMSCs remain unclear. In this study, we suggest that enhanced apoptosis and suppressed adipogenesis by capsaicin may contribute to fat removal in differentiating BMSCs.

\section{MATERIALS AND METHODS}

\section{Adipocyte differentiation and capsaicin exposure}

The BMSCs were kindly provided by the Cell and Tissue Biotechnology lab of Korea University and were cultured in Dulbecco's modified Eagle's medium (Gibco, USA) supplemented with $10 \%$ fetal bovine serum, $1 \%$ penicillin-streptomycin (Cambrex, Walkersville, MD, USA) and $2 \mathrm{mM} \mathrm{L}$-glutamine (Welgene, Daegu, Korea) at $37^{\circ} \mathrm{C}$ in a humidified $5 \% \mathrm{CO}_{2}$ incubator. When the cells reached confluence, adipogenic differentiation was induced by the addition of adipogenic agents $(10 \mu \mathrm{g} / \mathrm{mL}$ insulin, $5 \mu \mathrm{M}$ dexamethasone, and $0.5 \mathrm{mM} 3$-isobutyl-1-methylxanthin) to the culture medium. Cells were treated with $10,50,100$, or $200 \mu \mathrm{M}$ capsaicin during adipogenic differentiation. The medium was changed every 2 days for a total of 6 days. Cells were harvested $0,2,4$, and 6 days after the initiation of differentiation.

\section{Measurement of cell viability}

We assessed the effect of capsaicin on the growth and viability of differentiating BMSCs. Cell viability was examined using the 3-(4,5-dimethylthiazol-2-yl)-2,5diphenyltetrazolium bromide (MTT) assay kit (Sigma Aldrich, St. Louis, MO, USA). The MTT assays were performed according to the methods proposed by Mosmann. BMSCs were seeded into 12-well plates at a density of $1 \times 10^{5}$ cells/well. After $24 \mathrm{~h}$, the culture medium was replaced with that containing $200-\mu \mathrm{L}$ serial dilutions (0 to $10 \mu \mathrm{M})$ of capsaicin, and the cells were incubated for 2,4 , and 6 days. The final concentration of the solvent was less than $0.1 \%$ in the cell culture medium. Culture solutions were then removed and replaced with $90 \mu \mathrm{L}$ culture medium. Ten microliters of a sterile, filtered MTT solution $(5 \mathrm{mg} / \mathrm{mL})$ in phosphate-buffered saline (PBS, $\mathrm{pH}=7.4)$ was added to each well to a final concentration of $0.5 \mathrm{mg}$ MTT $/ \mathrm{mL}$. After $5 \mathrm{~h}$, the unreacted dye was removed, and the insoluble formazan crystals were dissolved in dimethyl sulfoxide (200 $\mu \mathrm{L} /$ well) and measured spectrophotometrically in a fluorescent microplate readers galaxy spectrophotometer (BMG Lab technologies Ltd., Ortenberg, Germany) at $570 \mathrm{~nm}$. The percentage (\%) of cell population 
growth was expressed relative to growth of the control, calculated as $\mathrm{A}_{570} \mathrm{~nm}$ (capsaicin)/ $\mathrm{A}_{570} \mathrm{~nm}$ (control). The growth inhibitory concentration $\left(\mathrm{IC}_{50}\right)$ was calculated as the capsaicin concentration that caused $50 \%$ inhibition of cell population growth compared with the untreated controls.

\section{Oil red $\mathrm{O}$ staining and quantification}

BMSCs treated with capsaicin were stained using oil red O. Briefly, cells were rinsed twice with PBS $(\mathrm{pH}=7.4)$ and fixed with $10 \%$ formalin in PBS for 20 min. Cells were washed with $60 \%$ isopropyl alcohol and stained with $2 \%$ (wt/vol) oil red $\mathrm{O}$ reagent (Sigma) for $30 \mathrm{~min}$ at room temperature. Cells were washed with isopropyl alcohol followed by repeated washes with distilled water. Staining was quantified by extracting the oil red O stain with $100 \%$ isopropyl alcohol, after which the absorbance was measured at $510 \mathrm{~nm}$. The number of total cells per field was determined based on fluorescence followed and the number of cells containing oil red $\mathrm{O}$ stained inclusions. Cells containing a visibly stained vacuole were considered positive for staining. Additionally, for each view the percentage of area showing positive staining was determined using Image J software.

\section{Measurement of apoptosis}

The BMSCs were seeded into 12-well plates $\left(1.5 \times 10^{5} /\right.$ well $)$. Cells were maintained in medium containing $10 \%$ fetal bovine serum and capsaicin at the appropriate concentrations. The apoptotic effect of capsaicin on differentiating BMSCs was evaluated using the Annexin V staining kit (Sigma Aldrich, St. Louis, MO, USA). Cells were harvested by trypsinization, and staining was performed according to the manufacturer's instructions. Flow cytometry was performed using the FACSCalibur flow cytometry system (BD Biosciences, San Jose, CA, USA).

\section{Terminal deoxynucleotidyl transferase dUTP nick end labeling assay}

To confirm the occurrence of cell death after capsaicin exposure, we used the In Situ Cell Death Detection Kit (Roche applied science, Indianapolis, IN, USA). The cells were stained to measure apoptosis, and all staining procedures were performed according to the manufacturer's instructions. Briefly, after incubation with the tunnel mixture, the cells were rinsed with PBS three times and embedded with anti-fade reagent. The cells were then observed by fluorescence microscopy at an excitation of $570 \mathrm{~nm}$ and emission of $720 \mathrm{~nm}$.

\section{RNA extraction and real-time polymerase chain reaction analysis}

The BMSCs were harvested at 0,2, 4, and 6 days after induction of adipogenic differentiation. Total RNA was extracted using TRIzol reagent (Molecular Research Center) according to the manufacturer's instructions. Total RNA was quantified based on the absorbance at $260 \mathrm{~nm}$, and the integrity of total RNA was analyzed using $1 \%$ agarose gel electrophoresis and ethidium bromide staining of the $28 \mathrm{~S}$ and $18 \mathrm{~S}$ bands. Total RNA $(2 \mu \mathrm{g})$ was reverse transcribed into cDNA using an iScript cDNA Synthesis kit (Bio-Rad, Hercules, CA, USA) according to the manufacturer's instructions. Real-time polymerase chain reaction (PCR) was performed as described previously (Jeong et al., 2013) using the QuantiTect SYBR Green realtime PCR Master Mix (Qiagen, Valencia, CA, USA) and 7500 Fast Sequence Detection System (Applied Biosystems, Foster City, CA, USA). All primers were designed by

Table 1. Primer sequences used to real-time polymerase chain reaction

\begin{tabular}{|c|c|c|c|c|}
\hline Gene name, symbol & GenBank ID & $5^{\prime} \rightarrow 3^{\prime}$ & Sequences & $\begin{array}{l}\text { Amplicon size } \\
\text { (bp) }\end{array}$ \\
\hline CCAAT/enhancer binding protein (C/EBP), alpha, CEBPA & NM_176784 & $\begin{array}{l}\text { Forward } \\
\text { Reverse }\end{array}$ & $\begin{array}{l}\text { tggacaagaacagcaacgag } \\
\text { ttgtcactggtcagctccag }\end{array}$ & 130 \\
\hline Fatty acid binding protein 4 , FABP4 & NM_174314 & $\begin{array}{l}\text { Forward } \\
\text { Reverse }\end{array}$ & $\begin{array}{l}\text { ggatggaaaatcaaccacca } \\
\text { gtggcagtgacaccattcat }\end{array}$ & 84 \\
\hline Peroxisome proliferator-activated receptor gamma, PPARG & BC_116098 & $\begin{array}{l}\text { Forward } \\
\text { Reverse }\end{array}$ & $\begin{array}{l}\text { aggatggggtcctcatatcc } \\
\text { gcgttgaacttcacagcaaa }\end{array}$ & 121 \\
\hline Stearoyl-CoA desaturase, SCD & AF_188710 & $\begin{array}{l}\text { Forward } \\
\text { Reverse }\end{array}$ & $\begin{array}{l}\text { cgcccttatgacaagaccat } \\
\text { tagttgtggaagectcacc }\end{array}$ & 80 \\
\hline BCL2-associated X protein, BAX & NM_173894 & $\begin{array}{l}\text { Forward } \\
\text { Reverse }\end{array}$ & $\begin{array}{l}\text { tctgacggcaacttcaactg } \\
\text { tcgaaggaagtccaatgtcc }\end{array}$ & 135 \\
\hline Caspase 3, CASP3 & NM_001077840 & $\begin{array}{l}\text { Forward } \\
\text { Reverse }\end{array}$ & $\begin{array}{l}\text { gacagtggtgctgaggatga } \\
\text { caggatccgttctttgcatt }\end{array}$ & 119 \\
\hline Glyceraldehyde-3-phosphate dehydrogenase, GAPDH ${ }^{1}$ & BC_102589 & $\begin{array}{l}\text { Forward } \\
\text { Reverse }\end{array}$ & $\begin{array}{l}\text { acccagaagactgtggatgg } \\
\text { ttgagctcagggatgacctt }\end{array}$ & 125 \\
\hline
\end{tabular}

CCAAT, cytosine-cytosine-adenosine-adenosine-thymidine; BCL2, B-cell lymphoma 2.

${ }^{1}$ Housekeeping gene. 
Integrated DNA Technologies (Coralville, IA, USA) based on the National Center for Biotechnology Information published sequences (Table 1). The $\Delta \Delta \mathrm{Ct}$ (delta delta $\mathrm{Ct}$ ) method was used to determine the relative fold changes in mRNA expression normalized to the housekeeping gene glyceraldehyde-3-phosphate dehydrogenase (GAPDH).

\section{Statistical analyses}

All data were expressed as means \pm standard error of the mean. Differences between control and treated groups were analyzed by analysis of variance using SAS software (SAS Institute, Cary, NC, USA). A multiple comparison of the means was performed using Duncan's multiple range tests. A $p$ value $<0.05$ was considered to reflect statistical significance. All experiments were performed in triplicate, with three replicates per experiment.

\section{RESULTS}

Cell viability after capsaicin treatment on differentiating bovine bone marrow mesenchymal stem cells

To assess whether capsaicin inhibited the cell growth of BMSCs, cells were treated with capsaicin $(0$ to $10 \mu \mathrm{M})$ and analyzed using the MTT assay. Overall, cell proliferation was not affected in a dose- or time-dependent manner by capsaicin (Figure 1). In addition, the percentage of cell viability was not reduced by capsaicin. At a concentration of $10 \mu \mathrm{M}$, capsaicin reduced cell growth up to approximately $7 \%$ when compared with the control at 4 and 6 days $(\mathrm{p}<0.05)$. Cell viability was similar at most concentrations, except at the highest concentration of capsaicin $(10 \mu \mathrm{M})$. Thus, we found that cell proliferation after capsaicin treatment was not suppressed in a dose- or time-dependent manner in differentiating BMSCs.
Effect of capsaicin on lipid deposition during bovine bone marrow mesenchymal stem cells differentiation

To further characterize the effects of capsaicin on adipogenic differentiation, the cells were examined using oil red $\mathrm{O}$ staining, and the lipids were quantified using a spectrophotometer. As shown in Figure 2A, we found that capsaicin reduced lipid deposition dose-dependently compared with non-exposed cells. General staining of the lipid droplets was observed at all capsaicin concentrations. However, the number of stained cells was decreased in 1 to $10 \mu \mathrm{M}$ capsaicin-treated BMSCs, irrespective of the culturing period, compared with the control (Figure 2A). The reduction was observed with $1 \mu \mathrm{M}$ capsaicin for all exposure times. The reduction was enhanced significantly at increasing capsaicin concentrations. For quantitative analysis, we confirmed that lipid deposition was reduced by capsaicin in differentiating BMSCs. The percentages of oil red $\mathrm{O}$ staining after $0,0.1,1,5$, and $10 \mu \mathrm{M}$ capsaicin treatment were $98.09 \%$ (control), 98.59\%, 87.76\%, 86.94\%, and $79.13 \%$ after 2 days; $99.32 \%, 99.40 \%, 92.75 \%, 89.62 \%$, and $59.41 \%$ after 4 days; and $99.63 \%, 99.52 \%, 89.97 \%$, $85.45 \%$, and $45.14 \%$ after 6 days, respectively (Figure 2B). Therefore, capsaicin suppressed adipocyte differentiation during longer culture periods and at higher concentrations.

Effects of capsaicin on the expression of adipogenesisand apoptosis-related genes

We examined whether capsaicin regulates the expression of adipogenesis- and apoptosis-related genes in differentiating BMSCs using real-time PCR. As shown in Figure 3, the mRNA expression of each gene was normalized to GAPDH, as the internal standard, and represented as the ratio relative to day 0 in each treatment.

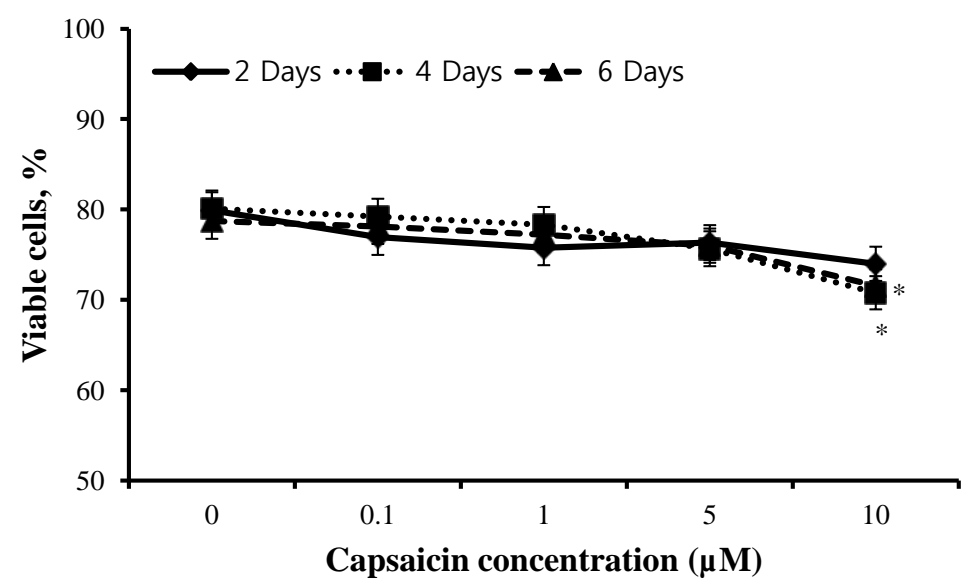

Figure 1. Effects of capsaicin on differentiating bovine bone marrow mesenchymal stem cells (BMSCs). Cell viability was determined using 3-(4,5-dimethylthiazol-2-yl)-2,5-diphenyltetrazolium bromide (MTT) assay. Our data demonstrate the dose- and time-dependent inhibitory effects of capsaicin $(10 \mu \mathrm{M})$. Values represent the means \pm standard error of the mean. $* \mathrm{p}<0.05$. The $\mathrm{x}$-axis indicates the concentration of capsaicin tested. The y-axis indicates the percentage of cell viability. Three experiments were performed for each treatment. 


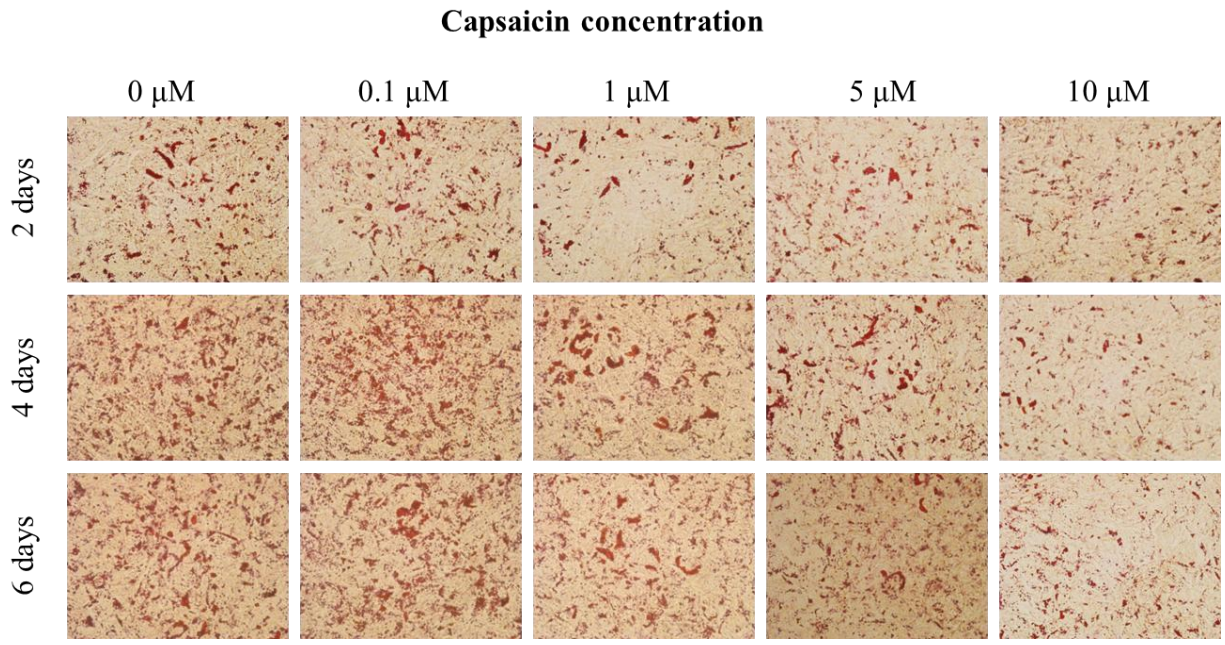

(A)

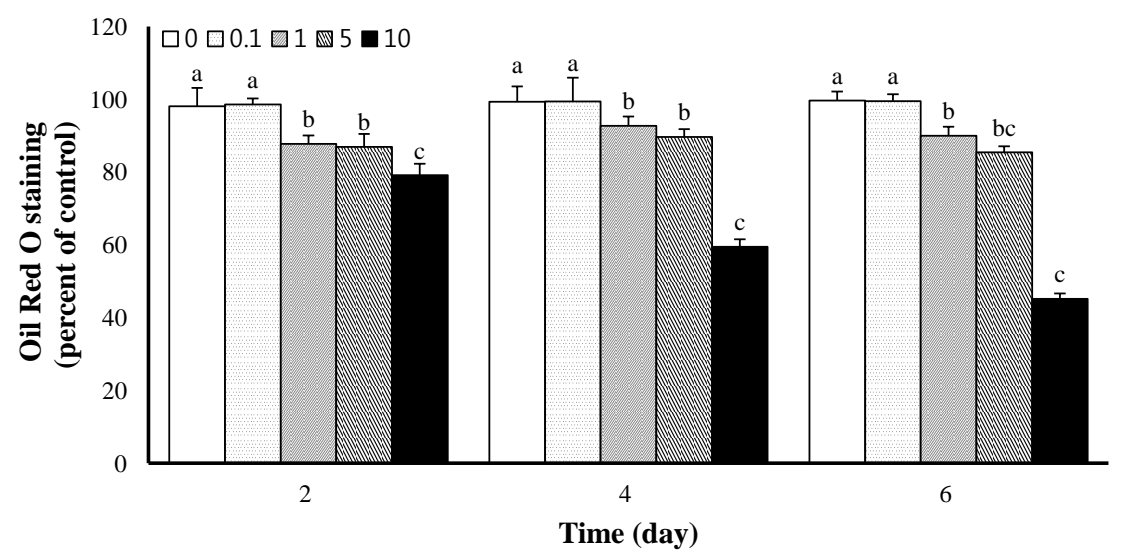

(B)

Figure 2. Fat depositions were examined using oil red $\mathrm{O}$ staining. Lipid droplets were reduced in cells treated with capsaicin. (A) visualization of lipid accumulation based on oil red $\mathrm{O}$ staining. Capsaicin reduced lipid deposition in a dose-dependent manner. (B) number of oil red O-stained cells per microscopic field $(n=3)$. Values represent the means \pm standard error of the mean. The different letters represent significant differences compared with untreated capsaicin $(0 \mu \mathrm{M})(\mathrm{p}<0.05)$. The $\mathrm{x}$-axis indicates the capsaicin exposure time. The y-axis indicates the percentage of oil red $\mathrm{O}$ stained cells.

Different adipogenic genes, such as PPARG, CEBPA, $F A B P 4$, and $S C D$, showed lower expression levels in the presence of capsaicin compared with the control (without capsaicin) ( $<<0.05$; Figure 3A). Adipogenic genes showed the lowest mRNA expression levels in the presence of capsaicin after 6 days. Expression levels of FABP4 and SCD were not significantly different after 2 or 4 days, respectively. In contrast, the expression of adipogenesisrelated genes decreased in differentiating BMSCs compared with the control after both 4 and 6 days. The effects of capsaicin on the expression of BAX and CASP3 are shown in Figure 3B. Capsaicin stimulated BAX and CASP3 expression significantly in dose- and time-dependent manners. The BAX expression increased at high concentrations of capsaicin $(\geq 5 \mu \mathrm{M})$ at 4 and 6 days compared with low concentrations $(\leq 1 \mu \mathrm{M})$ and the control $(\mathrm{p}<0.05$; Figure 3B). Compared with the control, BAX was upregulated maximally to 1.64 -fold $(\mathrm{p}<0.05)$ after 6 days but was not significantly different after 2 days. The transcription level of BAX at day 2 did not differ significantly among the experimental groups. The transcription level of CASP3 was similar to that of BAX in all treatment groups. However, the expression levels were not significantly different after 4 days. The CASP3 showed higher expression levels at $5 \mu \mathrm{M}$ capsaicin for all treatment periods.

\section{Effect of capsaicin on apoptosis during bovine bone marrow mesenchymal stem cells differentiation}

As described above, we found that lipid deposition was reduced and the adipogenesis- and apoptosis-related genes regulated by capsaicin. Thus, we next investigated whether the reduction in cell number by capsaicin exposure was due to apoptosis. Cell death was detected using the Terminal 
(A) Adipogenic genes
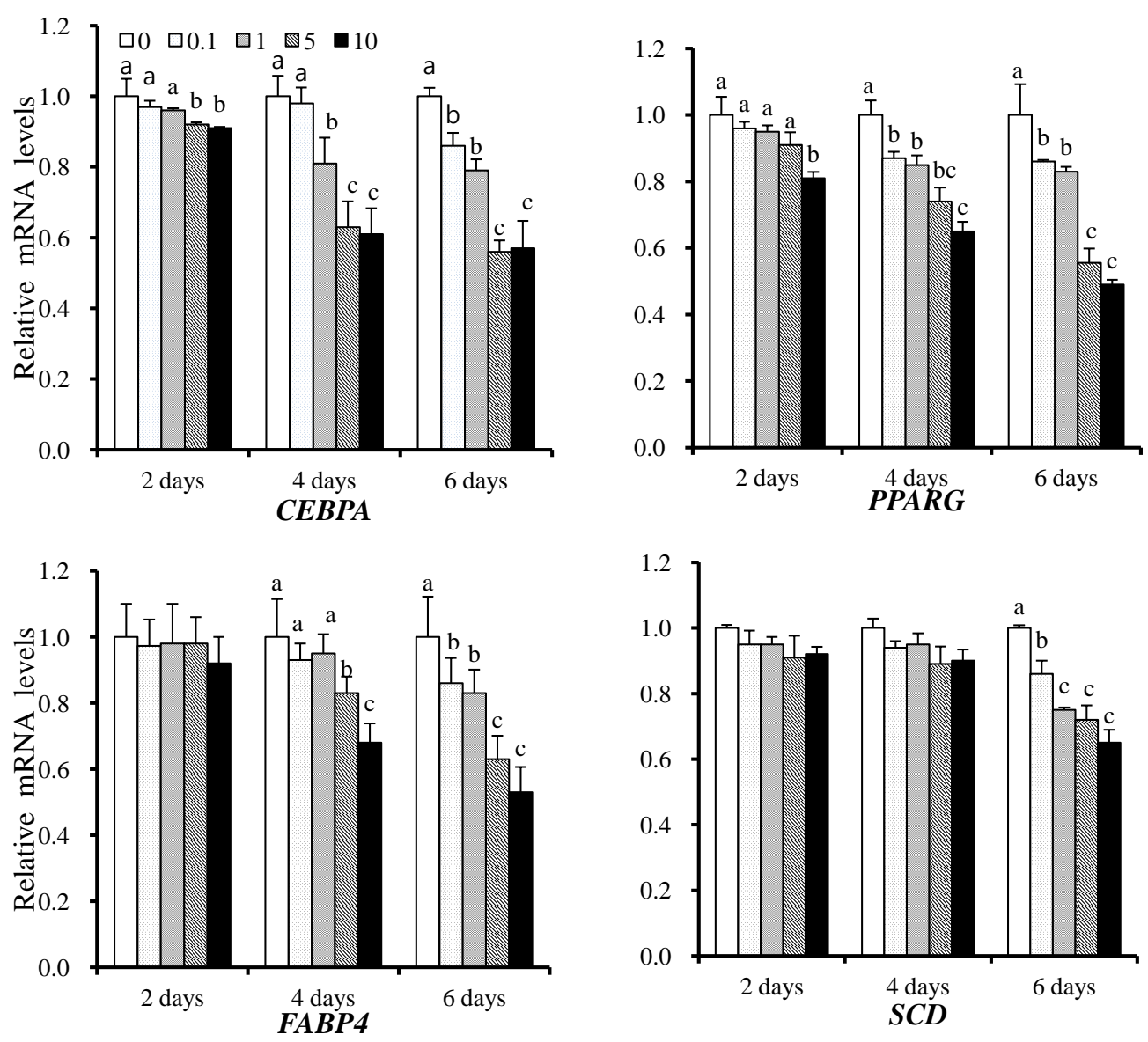

(B) Apoptotic genes
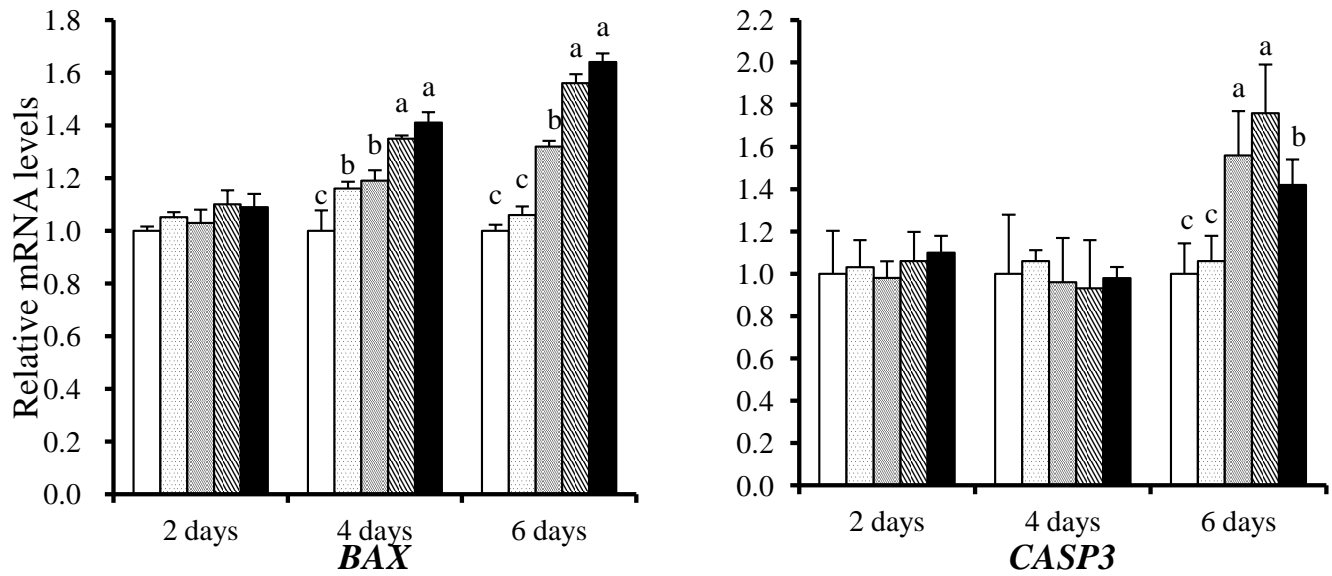

Figure 3. mRNA expression levels of adipogenic and apoptotic genes among various capsaicin-exposed groups. (A) mRNA expression levels of PPARG, CEBPA, FABP4, and SCD were decreased in differentiating bovine bone marrow mesenchymal stem cells (BMSCs) exposed to capsaicin. (B) apoptotic genes such as BAX and CASP3 were increased by capsaicin. mRNA levels were determined using real-time PCR and normalized to the housekeeping gene, GAPDH. The mRNA levels in untreated capsaicin cells at each time point were set to 1.0. Values represent the means \pm standard error of the mean. The different letters represent significant differences compared with untreated cells $(\mathrm{p}<0.05)$. PPARG, Peroxisome proliferator-activated receptor gamma; CEBPA, cytosine-cytosine-adenosine-adenosinethymidine/enhancer binding protein (C/EBP), alpha; FABP4, fatty acid binding protein 4; SCD, stearoyl-CoA desaturase; BAX, B-cell lymphoma 2-associated X protein; CASP3, Caspase 3; GAPDH, glyceraldehyde-3-phosphate dehydrogenase; PCR, polymerase chain reaction. 


\section{Capsaicin concentration}

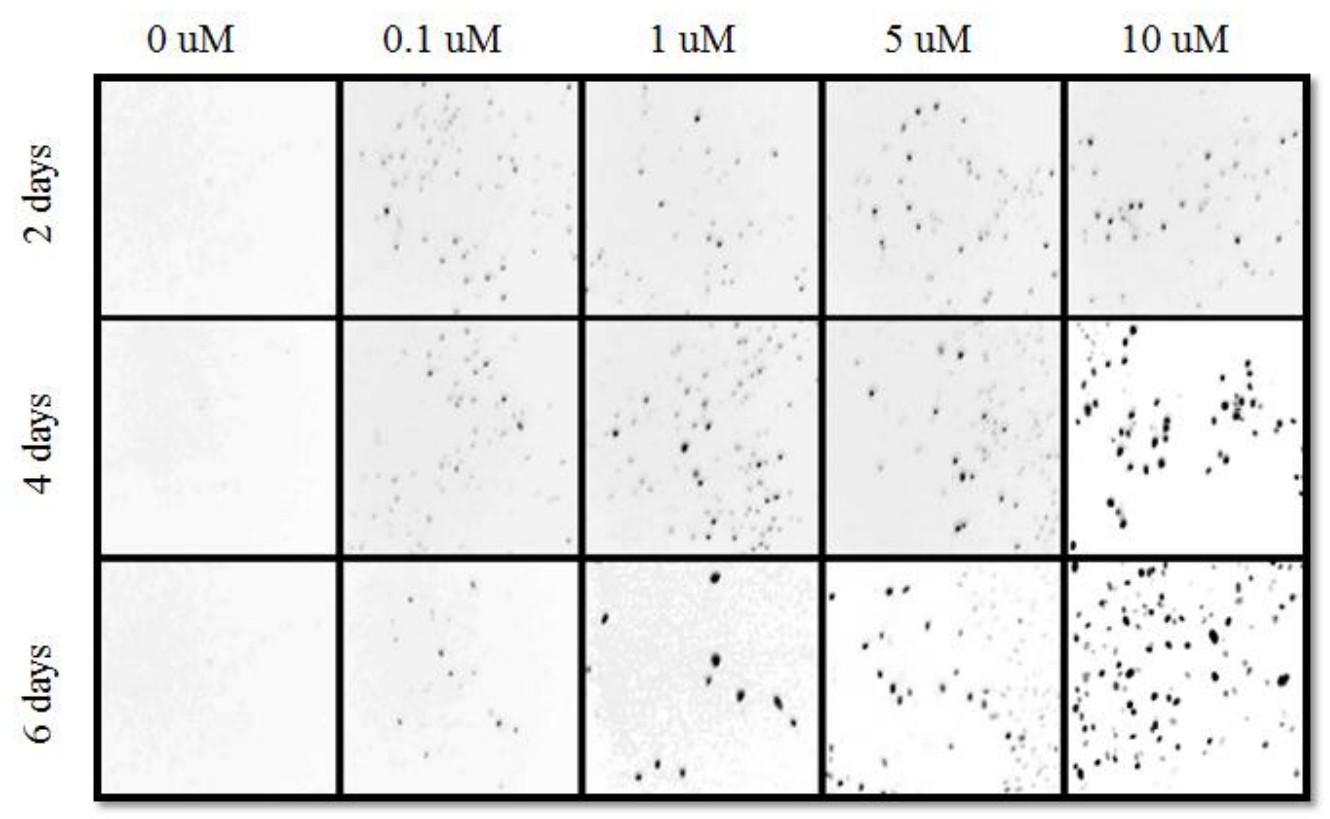

Figure 4. Terminal deoxynucleotidyl transferase dUTP nick end labeling (TUNEL) assay was performed to detect cell death among various capsaicin-exposed bovine bone marrow mesenchymal stem cell (BMSC) groups. Representative TUNEL assay images in the cells are shown $(\times 100)$. First row: capsaicin treatment for 2 days, second row: capsaicin treatment for 4 days, third row: capsaicin treatment for 6 days. Cell death caused by capsaicin exposure was indicated by red coloring. BMSCs were stained with fluoresceinlabeled dUTP according to the protocols described above.

deoxynucleotidyl transferase dUTP nick end labeling (TUNEL) assay at various capsaicin concentrations and exposure times. TUNEL staining increased in dose- and time-dependent manners in differentiating BMSCs (Figure 4). Therefore, cell death may be caused by different mechanisms such as apoptosis and necrosis. The TUNEL method is a common technique used to analyze apoptosis and necrosis. However, this assay could not distinguish between different types of cell death. To evaluate apoptosis specifically, fluorescence-activated cell sorting was performed in differentiating BMSCs using Annexin V/PI staining. According to the results, capsaicin may be associated with cell viability and the induction of early or late apoptosis rather than necrosis (Figure 5A). The percentage of apoptosis generally increased in the presence of capsaicin in dose- and time-dependent manners (Figure 5B). The number of apoptotic cells increased significantly (maximum of 33.6\%) after $10 \mu \mathrm{M}$ capsaicin exposure for 6 days $(\mathrm{p}<0.05)$. These results showed that apoptosis was correlated with cell viability and increased after treatment with capsaicin, compared with necrosis.

\section{DISCUSSION}

We previously examined the effects of epigallocatechin3-gallate (EGCG) in tea on anti-adipogenesis and apoptosis in BMSCs. Phytochemicals such as ECCG and capsaicin affect adipocyte differentiation and apoptosis in 3T3-L1 and stem cells (Hsu and Yen, 2007; Shin et al., 2009; Lee et al., 2011). Moreover, capsaicin reduces fat deposition in high fat-fed rats (Pande and Srinivasan, 2012). However, the effects of capsaicin in apoptotic cell death and adipogenesis of BMSCs remain unclear. Thus, the purpose of this study was to investigate the in vitro effects of capsaicin during adipogenic differentiation of BMSCs. Overall, capsaicin suppressed adipogenic differentiation and induced apoptosis in BMSCs.

Although BMSCs are derived from bone marrow, they can differentiate into mature adipocytes. Thus, we focused on the effects of capsaicin on induction of apoptosis and reduction of lipid accumulation during adipogenic differentiation. The concentrations of capsaicin used in this study were described previously (Hwang et al., 2005; Hsu and Yen, 2007). Here, we clearly indicated that capsaicin inhibited the growth of BMSCs. Previous studies showed that capsaicin decreased adipogenesis and lipolysis, and decreased cell viability in 3T3-L1 cells (Hwang et al., 2005; Hsu and Yen, 2007; Lee et al., 2011). Capsaicin affects cell proliferation and viability in tumor cells (Skrzypski et al., 2014). We found that capsaicin generally suppressed cell proliferation in dose- and time-dependent manners in differentiating BMSCs.

Lipid accumulation during adipocyte differentiation is regulated by capsaicin (Hwang et al., 2005; Zhang et al., 


\section{Capsaicin concentration}

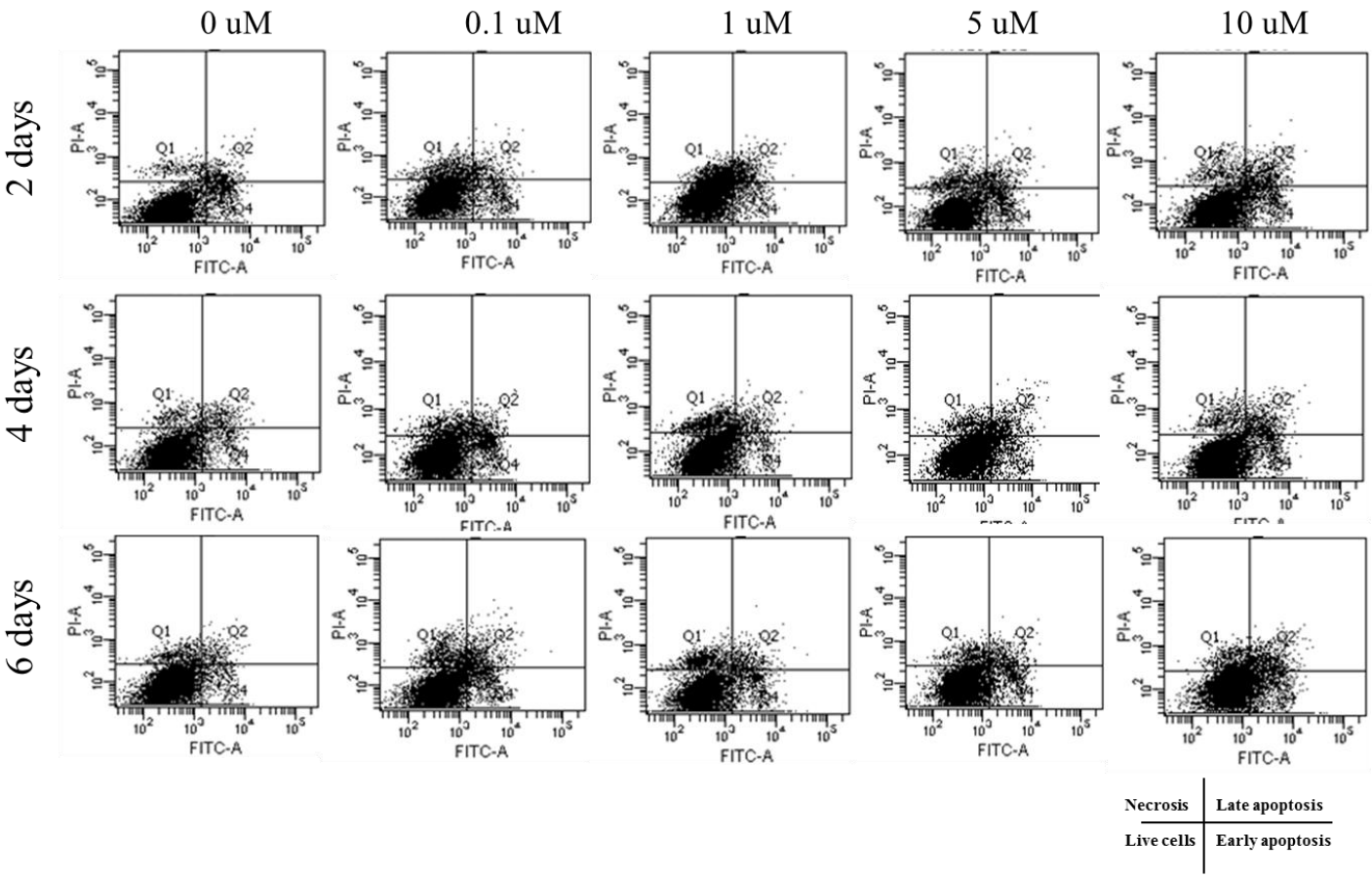

(A)

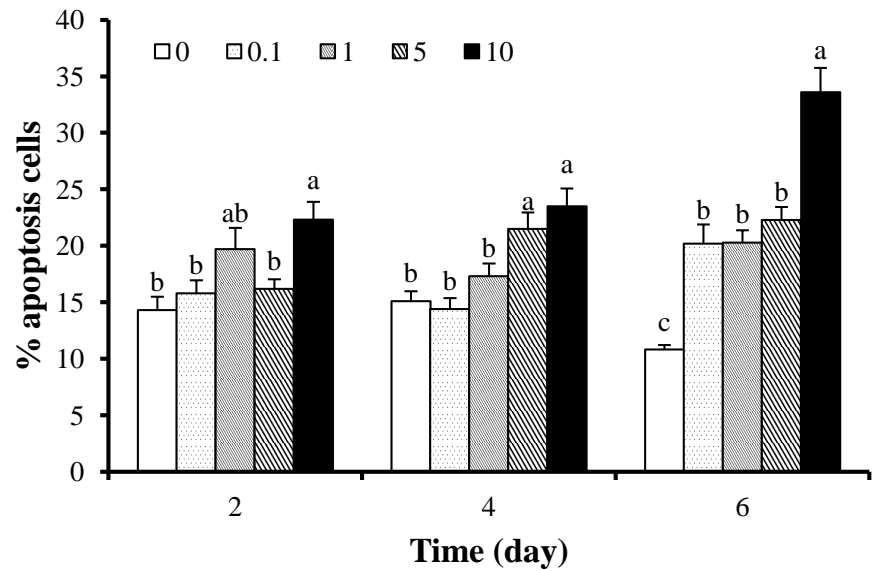

(B)

Figure 5. Flow cytometric analysis among various capsaicin-exposed differentiating BMSC groups. (A) the histogram indicates dose-and time-dependent reduction of apoptosis with concomitant increases in annexin V-positive cells. Cell cycle was examined using PI staining. Apoptosis was analyzed using annexin V staining. PI-A, absorbance for propidium iodide; FITC-A, absorbance for fluorescein isothiocyanate (fluorescein isothiocyanate-Annexin V). Q1, necrosis; Q2, late apoptosis; Q3, live cells; Q4, early apoptosis. (B) the percentages of apoptotic cells in the presence of capsaicin at different doses and for varying times are shown. These results suggest that capsaicin treatment led to cell cycle arrest and apoptosis in time- and dose-dependent manners. Values represent the means \pm standard error of the mean. The different letters represent significant differences compared with the control $(\mathrm{p}<0.05)$. The $\mathrm{x}$-axis indicates the capsaicin exposure time. The y-axis indicates the percentage of apoptotic cells.

2007). In addition, fat drops are inhibited in dose- and timedependent manners by capsaicin. Hepatic stellate cells treated with capsaicin showed decreased lipid accumulation in dose- and time-dependent manners (Bitencourt et al., 2012). Phytochemicals affect the morphology of lipid droplets, and capsaicin may modulate lipid accumulation and act as a lipid reducer in obese models. Therefore, we also showed that adipocyte differentiation was significantly downregulated by capsaicin. Moreover, the effects of capsaicin on expression of adipogenesis- and apoptosisrelated genes are shown here, as described above.

The PPARG is a key adipogenic transcription factor that plays an important role in adipogenesis and fatty acid metabolism. This nuclear transcription factor is highly 
expressed in obese animals, and its activity affects differentiation pathways in hepatic stellate and 3T3-L1 cells through several mechanisms (Tsukamoto et al., 2006; Guimarães et al., 2007; Hsu and Yen, 2007). Furthermore, the activation of transcription is required for inhibiting cell proliferation via apoptosis and suppressing extracellular matrix gene expression and lipid storage capacity (Wang et al., 2011; Zhang et al., 2011). Capsaicin may have PPARG ligand-like activity (Kim et al., 2004; Park et al., 2014). Capsaicin decreases energy intake and adipose tissue weight and inhibits the growth of various immortalized cell lines (Haramizu et al., 2011). Furthermore, it regulates various enzymes related to lipid catabolism, such as acetylCoA carboxylase and fatty acid synthesis. Similarly, Hwang et al. (2005) reported that capsaicin decreased the expression of adipogenic-specific genes significantly during adipocyte differentiation in 3T3-L1 cells. Adipocyte differentiation decreased the expression levels of FABP4 and CEBPA in these cells (Lee et al., 2011; Bitencourt et al., 2012). The SCD is also important for the formation of double bonds during de novo fatty acid synthesis (Beswick and Kennelly, 2000). Another study showed that capsaicin stimulated apoptosis of Swiss albino mice via CASP3 induction (Anandakumar et al., 2013). Caspases are essential for apoptotic cell death. To examine the lipid mechanisms underlying capsaicin-induced apoptosis of BMSCs, mRNA levels of various key apoptosis-related genes, including CASP3 and BAX, were measured. Our data also showed that capsaicin-induced apoptosis is controlled by the activation of CASP3 and BAX. The CASP3 is a candidate cell death-inducing protease that cleaves poly (ADP-ribose) polymerase (Chang and Yang, 2000). Moreover, capsaicin can modulate apoptosis in tumor cells via caspase, BCL-2, and BAX expression. These early results and our data suggest that capsaicin inhibits adipogenesis and induces apoptosis. Capsaicin and EGCG inhibit adipogenic differentiation by regulating adipogenic genes; however, their effects on cell apoptosis were not associated with the regulation of apoptotic genes, such as BAX and CASP3. In this study, we showed that the concentration of capsaicin $(\geq 1 \mu \mathrm{M})$ inhibits lipid accumulation and decreases the expression of adipogenic genes such as CEBPA, FABP4, PPARG, and SCD. We also found that BAX and CASP3 expression increased in the presence of capsaicin. Capsaicin regulation has previously been associated with anti-adipogenesis during differentiation (Bitencourt et al., 2012; Tang and Lane, 2012).

Capsaicin-mediated cell death (apoptosis or necrosis) increases in dose- and time-dependent manners according to flow cytometry (Hsu and Yen, 2007). Capsaicin induced apoptosis through a p53-dependent mechanism, ROS, and caspase- 3 activation. Capsaicin can induce apoptosis, which is important in maintaining cellular homeostasis and tissue development. Thus, fat metabolism in animals can be controlled by adipocyte apoptosis. Early apoptosis can be detected using flow cytometry (Vermes et al., 1995). Capsaicin induces cell apoptosis by activating ROS-induced DNA damage and BAX (Chakraborty et al., 2014). The EGCG and capsaicin stimulated intracellular ROS release, ultimately leading to cell death (Hwang et al., 2005). Therefore, our results showed that BMSCs treated with capsaicin increased apoptotic cell death versus necrosis in a dose-dependent manner. In this study, we found that capsaicin was actively involved in the induction of apoptosis during both the early and late stages of adipogenesis based on Annexin V and TUNEL staining.

In conclusion, capsaicin induced apoptosis by activating CASP3 and BAX, and it decreased mRNA expression of adipogenesis-related genes such as PPARG, CEBPA, FABP4, and $S C D$. Our results suggest that the combined effects of enhanced apoptosis and suppressed adipogenesis by capsaicin may contribute to fat deposition in differentiating BMSCs.

\section{ACKNOWLEDGMENTS}

This study was supported by the Postdoctoral Fellowship Program (Project No: PJ008487) from the National Livestock Research Institute and by a grant from the Next-Generation BioGreen 21 Program (Project No: PJ00819101) of Rural Development Administration, Republic of Korea.

\section{REFERENCES}

Anandakumar, P., S. Kamaraj, S. Jagan, G. Ramakrishnan, and T. Devaki. 2013. Capsaicin provokes apoptosis and restricts benzo(a)pyrene induced lung tumorigenesis in Swiss albino mice. Int. Immunopharmacol. 17:254-259.

Beswick, N. S. and J. J. Kennelly. 2000. Influence of bovine growth hormone and growth hormone-releasing factor on messenger RNA abundance of lipoprotein lipase and stearoylCoA desaturase in the bovine mammary gland and adipose tissue. J. Anim. Sci. 78:412-419.

Bitencourt, S., F. C. de Mesquita, E. Caberlon, G. V. da Silva, B. S. Basso, G. A. Ferreira, and J. R. de Oliveira. 2012. Capsaicin induces de-differentiation of activated hepatic stellate cell. Biochem. Cell Biol. 90:683-690.

Chakraborty, S., M. Mazumdar, S. Mukherjee, P. Bhattacharjee, A. Adhikary, A. Manna, S. Chakraborty, P. Khan, A. Sen, and T. Das. 2014. Restoration of $\mathrm{p} 53 / \mathrm{miR}-34 \mathrm{a}$ regulatory axis decreases survival advantage and ensures Bax-dependent apoptosis of non-small cell lung carcinoma cells. FEBS Lett. 588:549-559.

Chang, H. Y. and X. Yang. 2000. Proteases for cell suicide: functions and regulation of caspases. Microbiol. Mol. Biol. Rev. 64:821-846. 
Deans, R. J. and A. B. Moseley. 2000. Mesenchymal stem cells: biology and potential clinical uses. Exp. Hematol. 28:875-884.

Guimarães, E. L. M., M. F. S. Franceschi, C. M. B. Andrade, R. M. Guaragna, R. Borojevic, R. Margis, E. A. Bernard, and F. C. R. Guma. 2007. Hepatic stellate cell line modulates lipogenic transcription factors. Liver Int. 27:1255-1264.

Haramizu, S., F. Kawabata, Y. Masuda, K. Ohnuki, T. Watanabe, S. Yazawa, and T. Fushiki. 2011. Capsinoids, non-pungent capsaicin analogs, reduce body fat accumulation without weight rebound unlike dietary restriction in mice. Biosci. Biotechnol. Biochem. 75:95-99.

Hsu, C. L. and G. C. Yen. 2007. Effects of capsaicin on induction of apoptosis and inhibition of adipogenesis in 3T3-L1 cells. J. Agric. Food Chem. 55:1730-1736.

Hwang, J. T., I. J. Park, J. I. Shin, Y. K. Lee, S. K. Lee, H. W. Baik, J. Ha, and O. J. Park. 2005. Genistein, EGCG, and capsaicin inhibit adipocyte differentiation process via activating AMPactivated protein kinase. Biochem. Biophys. Res. Commun. 338:694-699.

Jeong, J., J. Bong, G. D. Kim, S. T. Joo, H. J. Lee, and M. Baik. 2013. Transcriptome changes favoring intramuscular fat deposition in the longissimus muscle following castration of bulls. J. Anim. Sci. 91:4692-4704.

Jeong, J., E. G. Kwon, S. K. Im, K. S. Seo, and M. Baik. 2012. Expression of fat deposition and fat removal genes is associated with intramuscular fat content in longissimus dorsi muscle of Korean cattle steers. J. Anim. Sci. 90:2044-2053.

Kennedy, A., K. Martinez, S. Schmidt, S. Mandrup, K. LaPoint, and M. McIntosh. 2010. Antiobesity mechanisms of action of conjugated linoleic acid. J. Nutr. Biochem. 21:171-179.

Kim, C. S., W. H. Park, J. Y. Park, J. H. Kang, M. O. Kim, T. Kawada, H. Yoo, I. S. Han, and R. Yu. 2004. Capsaicin, a spicy component of hot pepper, induces apoptosis by activation of the peroxisome proliferator-activated receptor gamma in HT29 human colon cancer cells. J. Med. Food 7:267-273.

Lee, M. S., C. T. Kim, I. H. Kim, and Y. Kim. 2011. Effects of capsaicin on lipid catabolism in 3T3-L1 adipocytes. Phytother. Res. 25:935-939.

Lin, C. H., W. C. Lu, C. W. Wang, Y. C. Chan, and M. K. Chen. 2013. Capsaicin induces cell cycle arrest and apoptosis in human KB cancer cells. BMC Complement. Altern. Med. 13:46.

Moisá, S. J., D. W. Shike, D. E. Graugnard, S. L. Rodriguez-Zas, R. E. Everts, H. A. Lewin, D. B. Faulkner, L. L. Berger, and J. J. Loor. 2013. Bioinformatics analysis of transcriptome dynamics during growth in angus cattle longissimus muscle. Bioinform. Biol. Insights 7:253-270.

Mosmann, T. 1983. Rapid colorimetric assay for cellular growth and survival: Application to proliferation and cytotoxicity assays. J. Immunol. Methods 65:55-63.

Pande, S. and K. Srinivasan. 2012. Potentiation of hypolipidemic and weight-reducing influence of dietary tender cluster bean (Cyamopsis tetragonoloba) when combined with capsaicin in high-fat-fed rats. J. Agric. Food Chem. 60:8155-8162.

Park, S. Y., J. Y. Kim, S. M. Lee, C. H. Jun, S. B. Cho, C. H. Park, Y. E. Joo, H. S. Kim, S. K. Choi, and J. S. Rew. 2014. Capsaicin induces apoptosis and modulates MAPK signaling in human gastric cancer cells. Mol. Med. Rep. 9:499-502.
Pittenger, M. F., A. M. Mackay, S. C. Beck, R. K. Jaiswal, R. Douglas, J. D. Mosca, M. A. Moorman, D. W. Simonetti, S. Craig, and D. R. Marshak. 1999. Multilineage potential of adult human mesenchymal stem cells. Science 284:143-147.

Ringe, J., C. Kaps, B. Schmitt, K. Buscher, J. Bartel, H. Smolian, O. Schultz, G. R. Burmester, T. Haupl, and M. Sittinger. 2002. Porcine mesenchymal stem cells. Induction of distinct mesenchymal cell lineages. Cell Tissue Res. 307:321-327.

Shin, D. W., S. N. Kim, S. M. Lee, W. Lee, M. J. Song, S. M. Park, T. R. Lee, J. H. Baik, H. K. Kim, J. H. Hong, and M. Noh. 2009. (-)-Catechin promotes adipocyte differentiation in human bone marrow mesenchymal stem cells through PPAR gamma transactivation. Biochem. Pharmacol. 77:125-133.

Simmons, D. J., P. Seitz, L. Kidder, G. L. Klein, M. Waeltz, C. M. Gundberg, C. Tabuchi, C. Yang, and R. W. Zhang. 1991. Partial characterization of rat marrow stromal cells. Calcif. Tissue Int. 48:326-334.

Skrzypski, M., M. Sassek, S. Abdelmessih, S. Mergler, C. Grötzinger, D. Metzke, T. Wojciechowicz, K. W. Nowak, and M. Z. Strowski. 2014. Capsaicin induces cytotoxicity in pancreatic neuroendocrine tumor cells via mitochondrial action. Cell. Signal. 26:41-48.

Tang, Q. Q. and M. D. Lane. 2012. Adipogenesis: From stem cell to adipocyte. Annu. Rev. Biochem. 81:715-736.

Prockop, D. J. 1997. Marrow stromal cells as stem cells for nonhematopoietic tissues. Science 276:71-74.

Tsukamoto, H., H. She, S. Hazra, J. Cheng, and T. Miyahara. 2006. Anti-adipogenic regulation underlies hepatic stellate cell transdifferentiation. J. Gastroenterol. Hepatol. 21(Suppl 3):S102-S105.

Vermes, I., C. Haanen, H. Steffens-Nakken, and C. Reutelingsperger. 1995. A novel assay for apoptosis. Flow cytometric detection of phosphatidylserine expression on early apoptotic cells using fluorescein labelled Annexin V. J. Immunol. Methods184:39-51.

Wang, C. Z., Y. Zhang, X. D. Li, Y. Hu, Z. G. Fang, D. J. Lin, R. Z. Xiao, R. W. Huang, H. Q. Huang, P. Q. Liu, and J. J. Liu. 2011. PPAR $\gamma$ agonist suppresses TLR4 expression and TNF- $\alpha$ production in LPS stimulated monocyte leukemia cells. Cell Biochem. Biophys. 60:167-172.

Wang, Y. W. and P. J. Jones. 2004. Conjugated linoleic acid and obesity control: efficacy and mechanisms. Int. J. Obes. Relat. Metab. Disord. 28:941-955.

Wise, L. S. and H. Green. 1979. Participation of one isozyme of cytosolic glycerophosphate dehydrogenase in the adipose conversion of ${ }_{3} \mathrm{~T}_{3}$ cells. J. Biol. Chem. 254:273-275.

Woodbury, D., E. J. Schwarz, D. J. Prockop, and I. B. Black. 2000. Adult rat and human bone marrow stromal cells differentiate into neurons. J. Neurosci. Res. 61:364-370.

Zhang, L. L., C. Y. Gao, C. Q. Fang, Y. J. Wang, D. Gao, G. E. Yao, J. Xiang, J. Z. Wang, and J. C. Li. 2011. PPAR $\gamma$ attenuates intimal hyperplasia by inhibiting TLR4-mediated inflammation in vascular smooth muscle cells. Cardiovasc. Res. 92:484-493.

Zhang, L. L., D. Y. Liu, L. Q. Ma, Z. D. Luo, T. B. Cao, J. Zhong, Z. C. Yan, L. J. Wang, Z. G. Zhao, S. J. Zhu, M. Schrader, F. Thilo, Z. M. Zhu, and M. Tepel. 2007. Activation of transient receptor potential vanilloid type-1 channel prevents adipogenesis and obesity. Circ. Res.100:1063-1070. 
Zhang, Y., N. Chen, J. Zhang, and Y. Tong. 2013. Hsa-let-7g miRNA targets caspase- 3 and inhibits the apoptosis induced by ox-LDL in endothelial cells. Int. J. Mol. Sci. 14:22708-22720.
Zuk, P. A., M. Zhu, H. Mizuno, J. Huang, J. W. Futrell, A. J. Katz, P. Benhaim, H. P. Lorenz, and M. H. Hedrick. 2001. Multilineage cells from human adipose tissue: Implications for cell-based therapies. Tissue Eng. 7:211-228. 